\title{
Destabilization of the Zhang-Rice singlet at optimal doping
}

\author{
D. K. Sunko \\ Department of Physics, Faculty of Science, University of Zagreb, \\ Bijenička cesta 32, HR-10000 Zagreb, Croatia.
}

December 4, 2018

\begin{abstract}
The construction of the Zhang-Rice singlet is revisited in the light of recent understanding of high-temperature superconductors at optimal doping. A minimal local model is derived which contains the physical regime found relevant for ARPES experiments, characterized by significant direct oxygen-oxygen hopping. For the values of orbital parameters indicated by experiment, the Zhang-Rice singlet is strongly mixed with a pure oxygen singlet of the same symmetry. The destabilization of the Zhang-Rice ground state is due to the oxygen singlet having twice as large a coherence factor with respect to oxygen-oxygen hopping. An analogous quantum phase transition is identified in the $t-t^{\prime}-J$ model. The orbital-antisymmetric copper-oxygen singlet is confirmed to be irrelevant, as found originally. The usual symmetry analysis is extended to include dynamical symmetries.
\end{abstract}

PACS: 74.72-h 74.25.Jb 71.10.Hf

\section{Introduction}

The nature of the electronic wave functions which undergo the transition to superconductivity in copper-oxide perovskites is still unknown. It is generally at least tacitly assumed that the superconducting (SC) transition at optimal doping does not invoke radically different states than those already present at the Fermi level slightly above $\mathrm{T}_{c}$. In this approach, shared here, the understanding of the normal state is considered the key to the SC mechanism.

The antiferromagnetic (AF) instability in the vicinity of half-filling in the same compounds is by contrast well understood [1] as a charge-transfer effect, dominating the first (large Mott-Hubbard interaction $U_{d}$ ) Coulomb correction 2 to the ideal (paramagnetic) Mott insulator [3]. Theoretical attempts to connect this underdoped region with the SC region on the hole-doped side abound in the literature. They have received early support in the ionic limit 
by the Zhang-Rice 4] construction, which considered an added hole in an AF background, when double occupation of the copper site was blocked by a large $U_{d}$. It is known that the hole then occupies the oxygen sites, each bridging two coppers in the $\mathrm{CuO}_{2}$ plane. Assuming that the hole prefers to bind with one of the coppers, Zhang and Rice have shown that a uniform spreading of the hole around that copper, in an orbitally symmetric "Zhang-Rice singlet" (ZRS) is energetically strongly preferred, and propagates like a projected fermion. This provided a way to vindicate the single-band $t-J$ model as a physical reduction of the Emery (three-band Hubbard) model [5, 6, at least for underdoped systems, as an alternative to the usual formal derivation, which applies the Foldy-Wouthuysen transformation to the one-band Hubbard model [7].

Challenges to this picture come from two directions. One is fundamental, that there is no a priori reason for the hole to prefer one copper over the other. When it is undecided, triplet states are strongly admixed, while it may still move about as a fermion [8. The other is to accept the basic idea of a single $\mathrm{CuO}_{4}$ molecule, but consider additional states and overlaps within it, to find the limits of the particular construction employed [9. In the present work, the natural next step in the Zhang-Rice construction is taken, which introduces just one additional singlet, occupying the oxygen sites.

As will be shown below, such an extension is the minimal model to express the low-energy physics of optimally doped high- $\mathrm{T}_{c}$ superconductors in the small-cluster language. At optimal doping, the Zhang-Rice ground state becomes strongly admixed with said oxygen singlet, chosen by the same symmetry arguments as originally used by Zhang and Rice [4].

The simplicity of the calculation is used as an opportunity to introduce classification of state vectors by dynamical symmetry on a small example. This method may have some potential for larger problems.

\section{Physical scales}

Quantum chemical calculations on small clusters predict the regime

$$
U_{d} \gg \Delta_{p d}>\left|t_{p d}\right| \gg\left|t_{p p}\right|,
$$

used by Zhang and Rice, and confirmed in XPS experiments [12]. Here $\Delta_{p d}=$ $\varepsilon_{p}-\varepsilon_{d}$ is the copper-oxygen splitting, positive in the hole picture, while $t_{p d}$ and $t_{p p}$ are the copper-oxygen and oxygen-oxygen overlaps, respectively. In the limit (1), the ZRS is the ground state. The energy scale of the XPS experiments and corresponding calculations is $5-10 \mathrm{eV}$. In high- $\mathrm{T}_{c}$ materials, there is a universally acknowledged crossover scale of the order of $0.1 \mathrm{eV}$ between the lowest and higher energies, although it is still debated whether its origin is phononic [13] or magnetic [14]. Neither small-cluster nor LDA calculations obtain this crossover, and the latter are consistent with the high-energy result (11). A recent analysis [15] showed three distinct many-body scales in BSCCO which were not reproduced by contemporary LDA calculations, the lowest of which was the above-mentioned crossover, the middle the so-called "waterfall," 
not of interest here, and the highest an "anomalous enhancement" of the LDApredicted band-width, by about $0.8 \mathrm{eV}$ visible below $\varepsilon_{F}$ in optimally doped $\mathrm{BSCCO}$, i.e. roughly twice that in full. In principle, LDA calculations are able to reach lower energy scales than small clusters. Thus no ab initio effort has so far reached the vicinity of the Fermi level in high- $\mathrm{T}_{c}$ superconductors. Below the crossover scale, the physical regime of the electrons actually undergoing the transition to superconductivity must still be inferred from phenomenological analysis of low-energy experiments.

The optimally doped region is equally well connected to the metallic overdoped region as to the underdoped one. Attempts to understand the high- $\mathrm{T}_{c}$ perovskites at optimal doping starting from the paramagnetic metal have a long standing on the hole-doped side [10. They have mostly been framed in terms of the mean-field slave-boson (MFSB) formalism, allowing for effective multiband approaches, in which the values of the orbital parameters depart significantly from those expected on the basis of high-energy experiments and LDA calculations [1].

Instead of taking the high-energy "bare" regime as the starting point, and introducing many-body effects to reach lower energies, MFSB phenomenology goes in the opposite direction. Beginning with unprejudiced zeroth-order fits at the Fermi level, one introduces the minimal perturbation necessary to model the lowest crossover. Such an analysis of angle-resloved photoemission (ARPES) [16] has recently been extended to electron-doped compounds [17, and produced the interesting result, that their lowest-energy sector is in a very similar physical regime as in the hole-doped ones [16, 18]: a large direct oxygen-oxygen hopping $t_{p p}$, significantly larger than the effective copper-oxygen hopping $t_{p d}$, and with a band-width of the same order as the effective copper-oxygen splitting $\Delta_{p d}>0$, in the hole picture:

$$
U_{d} \gg 4\left|t_{p p}\right| \gtrsim \varepsilon_{p}-\varepsilon_{d} \equiv \Delta_{p d} \gg t_{p d}^{2} / \Delta_{p d} .
$$

The ARPES scales on which this observation is based are $0.2 \mathrm{eV}$ in BSCCO and $0.5 \mathrm{eV}$ in NCCO. The lowest-energy crossover was modelled by a $(\pi, \pi)$ boson, whose scales were associated with AF in the same compounds. The low-energy regime (2), markedly different from (11), is characterized in particular by an "anticrossing" of the wide oxygen band with the copper level, so that the Fermi level is found in an oxygen-dominated, strongly dispersive part of the Brillouin zone, even though $\varepsilon_{d}<\varepsilon_{p}$. As will now be shown, a directly analogous regime exists in the ionic limit.

\section{Model and technique}

The Hamiltonian of a single $\mathrm{CuO}_{4}$ molecule is

$$
H=\varepsilon_{d} \sum_{\sigma} \hat{n}_{d \sigma}+\varepsilon_{p} \sum_{\sigma, i=1}^{4} \hat{n}_{p, i \sigma}
$$




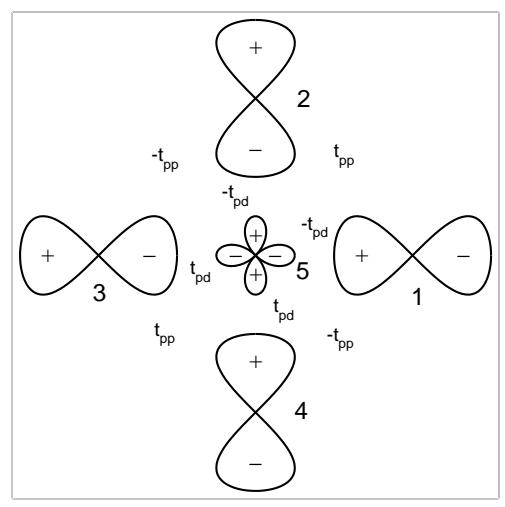

Figure 1: Orbital parameters and symmetries used in the article. In this sign convention $t_{p p}<0$ is physical [19].

$$
\begin{aligned}
& +t_{p d} \sum_{\sigma}\left(d_{\sigma}^{\dagger} P_{\sigma}+P_{\sigma}^{\dagger} d_{\sigma}\right)+U_{d} \hat{n}_{d \uparrow} \hat{n}_{d \downarrow} \\
& +t_{p p} \sum_{\sigma}\left(p_{+\hat{x}, \sigma}^{\dagger} p_{+\hat{y}, \sigma}+p_{-\hat{x}, \sigma}^{\dagger} p_{-\hat{y}, \sigma}+c . c .\right) \\
& -t_{p p} \sum_{\sigma}\left(p_{-\hat{x}, \sigma}^{\dagger} p_{+\hat{y}, \sigma}+p_{-\hat{y}, \sigma}^{\dagger} p_{+\hat{x}, \sigma}+c . c .\right),
\end{aligned}
$$

where $P_{\sigma}=p_{-\hat{x}, \sigma}+p_{-\hat{y}, \sigma}-p_{+\hat{x}, \sigma}-p_{+\hat{y}, \sigma}$ describes the four oxygens around the copper, and $\hat{n}_{d \sigma}, \hat{n}_{p, i \sigma}$ are the number operators of the copper and oxygen orbitals. The relevant orbitals and parameters [19] are shown in Figure 1] All 25 states of two holes with opposite spins on the five orbitals are taken into account.

Standard symmetry analysis is used to reduce the Hamiltonian (3) to blockdiagonal form. The basic symmetry is of the square, corresponding to the group $D_{4}$. Take the $z$-axis, orthogonal to the molecular plane, to be the spin quantization axis. Since the spins have total projection zero, flipping all spins is a symmetry operation, and since all orbitals are planar, this is equivalent to a reflection in the plane. Then $D_{4 h}$ is the magnetic (color) group of the problem, and both the Zhang-Rice singlet, $|Z R\rangle$, and the doubly-occupied copper state, $\left|d^{8}\right\rangle$, fall in its $A_{1 u}$ representation, containing five singlets.

As usual, the main symmetry group does not fix the state vectors in full. It is easy to complete the reduction by hand, but instructive to identify the reasons for the remaining ambiguities. One is, as expected, symmetry with respect to a subgroup, $D_{2 h} \subset D_{4 h}$. The particular $D_{2 h}$ group involved contains the spin-flip and three $180^{\circ}$ rotations, about the $z$-axis and about the two diagonals at $\pm 45^{\circ}$ relative to the $\mathrm{Cu}-\mathrm{O}$ axes. It stands out because its operations change the sign of the copper-oxygen overlap $t_{p d}$, while the energies depend only on $t_{p d}^{2}$.

The more interesting reason is dynamical symmetry, imposed by the assumed degeneracy of the four oxygen orbitals. It eventually affects only two 
$B_{1 u}$ vectors, but to fix them "honestly," the full dynamical symmetry apparatus must be invoked, even on such a small problem as the present one. The group elements are the Hamiltonian terms which break the oxygen degeneracy. Two are visible in (3), proportional to $+t_{p p}$ and $-t_{p p}$, respectively. If these are represented by matrices in one-particle space, multiplying them generates the third, which corresponds physically to next-nearest-neighbor (n-n-n) hops from one oxygen to the other on the same axis. These three (and the identity) form the Abelian group $D_{2}$, appearing here as a dynamical symmetry group. The n-n-n term must be diagonalized simultaneously with the sum of $t_{p p}$ terms in (3) to separate out the last vector without manual intervention.

The outstanding physical feature of dynamical symmetries is that they can connect states with doubly and singly occupied degenerate sites, obviously impossible by geometrical transformations. Two technical advantages of dynamical over geometrical symmetry groups are that they grow with the size of the problem, and depend on topology rather than shape. Both are significant when the clusters are motivated by solid-state investigations, and contain many equivalent sites. E.g., the dynamical group of the four oxygens in two-particle space is $Z_{2}^{4}$, with sixteen elements, again Abelian. It is not needed here because the geometrical symmetry already reduces the problem to very small blocks. On the other hand, as a molecule becomes larger, the blocks due to dynamical symmetry are expected to become smaller than those selected by geometrical symmetry, especially if the molecule is of odd shape, as is common in Monte Carlo calculations.

\section{Result}

The complete reduction gives ten stationary states unaffected by $t_{p d}$. In addition the Hamiltonian contains six $2 \times 2$ blocks and one $3 \times 3$ block. The latter is found in the $A_{1 u}$ subspace, connecting the states $\left|d^{8}\right\rangle,|Z R\rangle$, and an oxygen two-hole singlet, $\left|p^{-2}\right\rangle$ :

$$
\begin{aligned}
\left|p^{-2}\right\rangle= & \frac{1}{4}(|1\rangle+|2\rangle+|3\rangle+|4\rangle \\
& +|12\rangle-|13\rangle-|14\rangle-|23\rangle-|24\rangle+|34\rangle) .
\end{aligned}
$$

where $|i\rangle=|i \uparrow\rangle|i \downarrow\rangle$ are doubly occupied states, and $|i j\rangle=|i \uparrow\rangle|j \downarrow\rangle-|i \downarrow\rangle|j \uparrow\rangle$ are antisymmetrized singly occupied states. The numbers $1-4$ refer to the four oxygens, as in Fig. 11, The block reads:

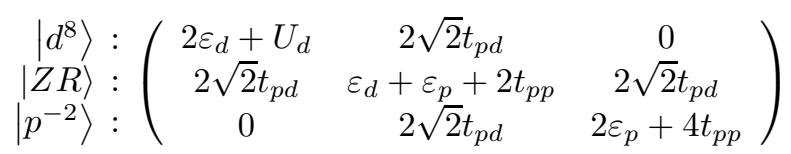

The states $\left|p^{-2}\right\rangle$ and $\left|d^{8}\right\rangle$ have an equally large coherence factor $2 \sqrt{2} t_{p d}$ with respect to $|Z R\rangle$, as found by perturbation theory [4. However, the coherence factor of the oxygen-oxygen overlap $t_{p p}$ is twice as large for $\left|p^{-2}\right\rangle$ as for $|Z R\rangle$. 


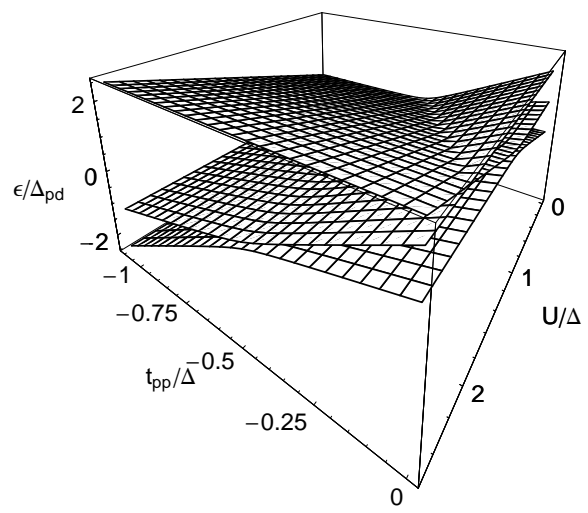

Figure 2: Parameter space of the model (5).

Since the physical sign of $t_{p p}$ is negative, this is a generic mechanism to destabilize the ZRS for all values of $U_{d}$ (including $U_{d} \rightarrow \infty$ ), which identifies $\left|p^{-2}\right\rangle$ as a relevant perturbation of the Zhang-Rice ground state.

By the same analysis, the orbital-antisymmetric singlet $|O A\rangle$, also considered by Zhang and Rice, appears in a $2 \times 2, B_{1 u}$ block with another oxygen singlet, call it $|p p\rangle$ :

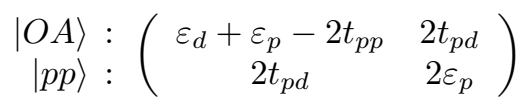

The splitting here is also $\Delta_{p d}+2 t_{p p}$, as between $\left|p^{-2}\right\rangle$ and $|Z R\rangle$ in (5), but it occurs because the copper-oxygen singlet is antibonding $\left(t_{p p}<0\right)$, while the oxygen singlet is non-bonding with respect to $t_{p p}$. Its structure is

$$
|p p\rangle=\frac{1}{2 \sqrt{2}}(-|1\rangle+|2\rangle-|3\rangle+|4\rangle+|13\rangle-|24\rangle) .
$$

The orbital-antisymmetric state remains irrelevant in the presence of oxygenoxygen hopping. The $t_{p d}$-coherence factors in (5) and (6) may be identified by perturbation theory [4, without the need to distinguish between $\left|p^{-2}\right\rangle$ and $|p p\rangle$. However, once $t_{p p}$ is introduced, the different effects on $|Z R\rangle$ and $|O A\rangle$ are related to the different symmetry properties of $\left|p^{-2}\right\rangle$ and $|p p\rangle$. The ZhangRice singlet $|Z R\rangle$ is destabilized because $\left|p^{-2}\right\rangle$ has a large coherence factor $4 t_{p p}$, while $|O A\rangle$ moves upwards "out of the way," and $|p p\rangle$ cannot influence the ground state because it is unaffected by $t_{p p}$ (but of course it can anticross with $|O A\rangle$ as the latter rises).

The coherence factors in the reduced model (5) are the largest in the whole space, so for $\left|t_{p d}\right|>0$ and $t_{p p}<0$ it contains the ground state. The relevant parameters are $U_{d} / \Delta_{p d}$ and $t_{p p} / \Delta_{p d}$. The precise value of $t_{p d}$ is irrelevant, as long as one is in the parameter regime (2), corresponding to optimal doping. Choosing it low for graphical reasons, one obtains Figure 2. At the origin $\left(U_{d}=0, t_{p p}=0\right)$ is the weak-coupling regime, where the ground state is 
dominated by $\left|d^{8}\right\rangle$. As $U_{d}$ increases, this state goes through two anticrossings with the others, ending up highest in the Zhang-Rice regime $\left(U_{d}\right.$ large, $\left|t_{p p}\right|$ small), where $|Z R\rangle$ is the ground state. Now as $\left|t_{p p}\right|$ increases, the state $\left|p^{-2}\right\rangle$ goes down at twice the rate of $|Z R\rangle$, so it must eventually anticross with it. Thus when both $U_{d}$ and $\left|t_{p p}\right|$ are large, the ZRS is no longer the ground state.

\section{Discussion}

The present paper describes a simple and robust symmetry-based mechanism which prevents the isolation of the ZRS as the only candidate for the ground state of the $\mathrm{CuO}_{4}$ ion when $U_{d}$ is large. To wit, if the oxygen-oxygen hopping $t_{p p}$ is significant, the local environment will be in a charge transfer regime involving two states, the ZRS and an oxygen singlet. The two are mixed by the symmetryenhanced overlap $2 \sqrt{2} t_{p d}$ across the symmetry-reduced gap $\Delta_{p d}+2 t_{p p}$. This is the metallic counterpart to the result [1] that the antiferromagnetic interaction $J$ near half-filling is also dominated by the charge-transfer term $t_{p d}^{4} / \Delta_{p d}^{3}$, while the Mott-Anderson contribution $t_{p d}^{2} / U$ is negligible.

It has long been established that ARPES fits require significant ratios of $\left|t_{p p} / t_{p d}\right|$ in order to agree with the data [11. Recently there appeared detailed ARPES fits, both qualitatively and quantitatively in accord with experiment, in practically the same orbital regime for $\mathrm{NCCO}$ [17] and BSCCO [16], which established the anticrossing regime (2) as an appropriate phenomenology for these compounds, meaning the ratio $\left|t_{p p}\right| / \Delta_{p d}$ is also enhanced, especially so on the electron-doped side.

Changes in orbital parameters as a function of doping are a standard feature of effective theories such as mean-field slave-boson (MFSB), which account for the effects of strong correlations by changing $t_{p d}$ and $\Delta_{p d}$. The question, as always with effective band models, is what this means for the local environment: do local hops "see" the bare or the renormalized band parameters. The conclusion that the effective band regime at optimal doping is reflected in an analogous local regime, which implies the destabilization of the Zhang-Rice singlet by the above symmetry mechanism, is supported by two independent arguments, one phenomenological and one theoretical.

The recently emphasized 15 failure of LDA calculations to reproduce experimentally observed band widths is phenomenologically significant, because LDA predicts a smaller width than is actually observed, so any conceivable inclusion of additional correlations would only make the discrepancy larger. Notably, LDA calculations agree with high-energy experiments and calculations in the ionic limit. The anomalous enhancement of hopping via the oxygen sites is consistent by symmetry with the observed discrepancy, which is large in the nodal direction, where the oxygen band is strongly dispersive. The enhancement of the ARPES bandwidth of 1-2 eV over LDA predictions [15] is quantitatively in good agreement with the value of $t_{p p}$ needed to enter the anticrossing regime, i.e. destabilize the ZRS.

Theoretically, the parameter space of the three-band MFSB model has been 
extensively investigated [18 from the point of view of outcomes, namely: which regions of renormalized orbital parameters are indicative of strong renormalization of input parameters by the MFSB mechanism, and which imply preservation of the bare parameters. In the so-called "resonant-band" regime, characterized by a narrow effective bonding band and copper-dominated wave functions, the quasiparticle weight in the conduction band is significantly reduced, being proportional to the doping $\delta$ instead of the total hole content $1+\delta$. In that case, the band calculations cannot be directly related to a local picture, since the doped-hole weight is locally either one or zero. However, in the anticrossing regime (2), the opposite occurs: MFSB theories with Gaussian fluctuations depart from the resonant-band regime and acquire a full quasiparticle weight in the Brillouin zone, only with a reduced $t_{p d}[18$. They are therefore comparable and consistent with the present ionic ones, as long as both are in the anticrossing limit. Thus the present work reaffirms the possibility [20] that the optimizations leading to a large direct oxygen-oxygen overlap below the crossover scale occur already at the local orbital level.

Realistically, $t_{p d}$ is not so small, so the ground state in real materials retains substantial copper content. In the local picture, this means a significant ZRS content in the ground state. However, even if $t_{p d}$ is as large as $\left|t_{p p}\right|$, giving a large anticrossing gap $\sim \Delta_{p d}$, the ground state still contains nearly equal components of $|Z R\rangle$ and $\left|p^{-2}\right\rangle$. The system is physically in the charge-transfer regime, which also provides the dominant contribution [1 to $J$. In that case, the reduction to one band by the limit [4] $\Delta_{p d}=U_{d}-\Delta_{p d} \rightarrow \infty$ is purely formal, since that limit replaces the charge-transfer regime a priori by the Mott regime.

While the MFSB calculations allow both for regimes with strong renormalization and no renormalization [18, the derivation of the $t-J$ model from the ZRS [4 implies by construction that the band parameters are locally relevant. However, it has been noticed [9] that in order to face experiment, the $t-J$ model needs to be supplemented by additional next- and next-next-nearest neighbor hops, making up the so-called $t-t^{\prime}-t^{\prime \prime}-J$ model. Since the $t^{\prime}$ term breaks particlehole symmetry in the same way as the $t_{p p}$ term in the three-band model, its appearance in practical calculations 21] is immediate evidence that the oxygen degree of freedom cannot be eliminated from the physical picture near optimal doping. The need to introduce $t^{\prime}$ at the band level translates microscopically into the need to admix the oxygen singlet (5) to the ZRS, to describe the local electronic environment around optimal doping. (When the oxygen is treated explicitly in the band picture, $t_{p p}$ has the same sign for both $\mathrm{n}$ - and p-doping [16, 17, as also found in LDA calculations [22], consistently with its simple chemical interpretation.)

To see the real effect of the $t^{\prime}$ term, the dispersion of the $t$ and $t^{\prime}$ terms may be rewritten

$$
\begin{aligned}
& -2 t\left(\cos k_{x}+\cos k_{y}\right)-4 t^{\prime} \cos k_{x} \cos k_{y}= \\
& \quad-4\left(t+t^{\prime}\right)+4\left(t+2 t^{\prime}\right)\left(\sin ^{2} \frac{k_{x}}{2}+\sin ^{2} \frac{k_{y}}{2}\right)-16 t^{\prime} \sin ^{2} \frac{k_{x}}{2} \sin ^{2} \frac{k_{y}}{2},
\end{aligned}
$$


separating contributions by particle-hole symmetry. The second term is manifestly symmetric around half-filling, while the third is not, since it achieves its minimum value (zero) along the non-dispersive lines $k_{x}=0$ and $k_{y}=0$. In practice $t t^{\prime}<0$, and the ratio $\left|t^{\prime} / t\right| \sim 0.2-0.4$. For $t^{\prime}=-0.3 t$, a common enough parametrization around optimal doping, $\left|4 t^{\prime} /\left(t+2 t^{\prime}\right)\right|=3$, so the symmetry-breaking term enters with three times the contribution of the symmetry-preserving term. Thus the apparently small values of $\left|t^{\prime} / t\right|$ found in the literature are quite misleading in terms of the actual amount of particle-hole symmetry breaking needed to achieve realistic Fermi surface fits within the $t-t^{\prime}-$ $J$ model. In particular, for $t^{\prime}=-t / 2$ the symmetry-preserving term vanishes, so the $t-t^{\prime}-J$ model passes through a quantum phase transition in the vicinity of its applicable parameter range.

By symmetry, this is the same type of transition appearing in the present paper as the destabilization of the ZRS in the three-band case. Physically, they are different insofar as the three-band model destabilizes the ZRS by oxygen hopping in low order, while in the $t-t^{\prime}-J$ model the Zhang-Rice singlets are preserved by assumption, and the transition occurs by the additional $t^{\prime}$ overlap among them, i.e. in high order in the original three-band model. But to account for ARPES data, both models agree that such a transition is approached near optimal doping.

The limitation of the above arguments to the metallic phase should be kept in mind. Near half-filling, the oxygen hopping is effectively reduced by the antiferromagnetic interactions, and precisely at half-filling, the system is insulating independently of the value of $t_{p p}$. In the insulating phase, the high-energy ionic limit is recovered, both theoretically and experimentally. As $t_{p p}$ becomes more effective with doping, locally the first admixture to the ZRS appears, identified here as the oxygen singlet (5). Thus a broad picture of the optimal-doping regimes of high- $\mathrm{T}_{c}$ compounds emerges, in which $\mathrm{NCCO}$ is deep in the anticrossing regime, $4\left|t_{p p}\right| \gg \Delta_{p d}$, and BSCCO is at its limit, $4\left|t_{p p}\right| \approx \Delta_{p d}$. On the other hand, the remarkable Fermi-surface evolution of LSCO with doping [23] is indicative of strong renormalizations of the band parameters, especially when the system is underdoped. In such a resonant-band regime, it was also shown directly 24] that the strong doping-dependence of $t_{p p}$ in the MFSB fits did not imply a similar variation of the local parameters.

The present analysis points to one pitfall of considering the oxygen part of the ZRS as a single-particle state in its own right, as if the hole on the copper were unhybridized with the oxygens. However, the same caution applies to the oxygen singlet found here. In particular, one should be careful in interpreting the corresponding Bloch states. The propagation of strongly correlated cluster states in a lattice is at present largely unexplored in more than one dimension, with controlled analytic results available only in special limits, which themselves amount to uncontrolled simplifications. Such is the limit of infinite dimensions 25, physically corresponding to diffusion, or the Falicov-Kimball limit [26], which is dispersive, but replaces dynamical constraints by geometrical ones.

The model presented here is as sparse as possible, allowing for the exper- 
imentally observed regime with only three state vectors. To avoid being too minimal, some attention should be paid to the neglected Coulomb integrals $U_{p}$, $V_{p p}$ and $V_{p d}$, the on-site oxygen, intracell oxygen, and copper-oxygen repulsions, respectively. They do not break the symmetries above, introducing only matrix elements within each symmetry block. In particular, they connect the groundstate block (5) with the other two $A_{1 u}$ states by matrix elements $U_{p} / 4$, smaller than the terms $2 \sqrt{2} t_{p d}$. On the diagonal of this block, the Coulomb terms can be reabsorbed in the site energies and copper on-site repulsion $U_{d}$. For realistic $V_{p p} \ll U_{p}$, this leads to a small reduction in the copper-oxygen splitting $\Delta_{p d}$, and practically no change in $U_{d}$, while for large $V_{p p}=U_{p} / 2$, a useful limiting case, the effective $U_{d}$ is increased and the effective $\Delta_{p d}$ decreased, both by about $1 \mathrm{eV}$. Thus the inclusion of neglected integrals mildly tends to push the system further into the anticrossing, $U_{d} \rightarrow \infty$ regime.

An extension of the Zhang-Rice argument to the electron-doped side is only possible with larger molecules, containing more than one copper atom. This would allow to study the competition of magnetic and charge ordering at the local level. It also opens a way to compare the Zhang-Rice and Emery-Reiter [8] constructions directly on the hole-doped side. Obviously, the spaces involved are much larger. It is on these larger problems, both theoretical and numerical, that hope is invested in the assignment of quantum numbers by dynamical symmetries, as introduced in the present work.

\section{Conclusion}

It has been found that in the presence of local repulsion on copper, the direct oxygen-oxygen overlap is a relevant perturbation of the Zhang-Rice singlet. With the possible exception of LSCO, real hole- and electron-doped high- $\mathrm{T}_{c}$ superconductors appear to be in a physical regime where it is strongly mixed with a pure oxygen singlet.

\section{Acknowledgments}

Conversations with S. Barišić are gratefully acknowledged. This work was supported by the Croatian Government under Project 119-1191458-0512.

\section{References}

[1] H. Eskes and J. H. Jefferson, Phys. Rev. B 48, 9788 (1993).

[2] P. W. Anderson, Phys. Rev. 115, 2 (1959).

[3] N. F. Mott, Proc. Phys. Soc. A 62, 416 (1949).

[4] F. C. Zhang and T. M. Rice, Phys. Rev. B 37, 3759 (1988). 
[5] V. J. Emery, Phys. Rev. Lett. 58, 2794 (1987).

[6] C. M. Varma, S. Schmitt-Rink, and E. Abrahams, Solid State Commun. 62, 681 (1987).

[7] P. Fulde, Electron correlations in molecules and solids, Springer, 1993.

[8] V. J. Emery and G. Reiter, Phys. Rev. B 38, 11938 (1988).

[9] A. M. Macridin, M. Jarrell, T. Maier, and G. A. Sawatzky, Phys. Rev. B $\mathbf{7 1}, 134527$ (2005).

[10] G. Kotliar, P. A. Lee, and N. Read, Physica C 153-155, 538 (1988).

[11] Qimiao Si, Yuyao Zha, K. Levin, and J. P. Lu, Phys. Rev. B 47, 9055 (1993).

[12] M. A. van Veenendaal, G. A. Sawatzky, and W. A. Groen, Phys. Rev. B 49, 1407 (1994).

[13] W. S. Lee, S. Johnston, T. P. Devereaux, and Z.-X. Shen, Phys. Rev. B 75, 195116 (2007).

[14] T. Valla et al., Phys. Rev. Lett. 98, 167003 (2007).

[15] W. Meevasana et al., Phys. Rev. B 75, 174506 (2007).

[16] D. K. Sunko and S. Barišić, Eur. Phys. J. B 46, 269 (2005).

[17] D. K. Sunko and S. Barišić, Phys. Rev. B 75, 060506(R) (2007).

[18] I. Mrkonjić and S. Barišić, Eur. Phys. J. B 34, 69 (2003).

[19] D. I. Golosov, A. E. Ruckenstein, and M. L. Horbach, J. Phys.: Condens. Matter 10, L229 (1998).

[20] A. A. Abrikosov and L. A. Fal'kovskii, JETP Letters 49, 531 (1989).

[21] B. Kyung, V. Hankevych, A.-M. Daré, and A.-M. S. Tremblay, Phys. Rev. Lett. 93, 147004 (2004).

[22] M. M. Korshunov et al., Phys. Rev. B 72, 165104 (2005).

[23] A. Ino et al., J. Phys. Soc. Jpn. 68, 1496 (1999).

[24] L. Janković and D. K. Sunko, Physica C 341-348, 2103 (2000).

[25] A. Georges, G. Kotliar, W. Krauth, and M. J. Rozenberg, Rev. Mod. Phys. 68, 13 (1996).

[26] D. K. Sunko, Eur. Phys. J. B 43, 319 (2005). 